\title{
Slow Decay of Impact in Equity Markets
}

\section{Brokmann}

Capital Fund Management

23, rue de l'Université

75007 Paris, France

\section{E. Sérié}

Capital Fund Management

23, rue de l'Université

75007 Paris, France

\section{J. Kockelkoren}

Capital Fund Management

23, rue de l'Université

75007 Paris, France

\section{J.-P. Bouchaud*}

Capital Fund Management

23, rue de l'Université

75007 Paris, France

jean-philippe.bouchaud@cfm.fr

Accepted 29 June 2015

Published 10 September 2015

Using a proprietary dataset of meta-orders and prediction signals, and assuming a quasi-linear impact model, we deconvolve market impact from past correlated trades and a predictable return component to elicit the temporal dependence of the market impact of a single daily meta-order, over a 10-day horizon in various equity markets. We find that the impact of single meta-orders is to a first approximation universal

*Corresponding author.

This is an Open Access article published by World Scientific Publishing Company. It is distributed under the terms of the Creative Commons Attribution 4.0 (CC-BY) License. Further distribution of this work is permitted, provided the original work is properly cited. 


\section{Brokmann et al.}

and slowly decays to zero (or to a small value), possibly as a power-law. We show that autocorrelated order-flows and trade information contents fully accounts for the apparent plateau observed in the raw data. We discuss the possible bias introduced by the quasi-linear assumption.

Keywords: Market impact; impact decay; equity markets; informed trades.

\section{Introduction}

One of the most fundamental question in financial economics is the shape and nature of price impact, to wit: How buying (/selling) a quantity $Q$ of shares (or contracts on future markets, or any other traded asset) will move the price, in particular when this quantity $Q$ is executed incrementally over time (Kyle, 1985; Bouchaud et al., 2008). There is now a remarkable consensus that the impact of such a meta-order — measured as the average difference between the final price and the initial price times the sign of the order — has an approximate square-root dependence on $Q$ (see, e.g. (Torre, 1997; Almgren et al., 2005; Moro et al., 2009; Tóth et al., 2011; Bladon et al., 2012; Kyle and Obizhaeva, 2012; Mastromatteo et al., 2014a; Zarinelli et al., 2014)). More precisely, a very large number of independent quantitative measures of price impact have concluded that to a first approximation, and independently of the asset class, time period, style of trading and micro-structure peculiarities, one has (see Fig. 2):

$$
\left\langle\epsilon \cdot \frac{p(T)-p(0)}{p(0)}\right\rangle \approx Y_{0} \sigma\left(\frac{Q}{V}\right)^{\delta} \equiv \theta,
$$

where $\epsilon= \pm 1$ for buy (sell) orders, $p(0)$ is the price at the start of the execution period and $p(T)$ is the price at the end of the execution period. Here and below, $\langle\ldots\rangle$ denote averaging. In the right-hand side of the equation, $\sigma$ and $V$ are, respectively, the volatility of the traded instrument and the total traded volume over the order execution time scale $T, Y_{0}$ is a dimensionless constant of order unity and $\delta$ is the impact exponent in the range $[0.4,0.7]$. Theoretical models allowing one to understand the mechanisms underlying this strongly concave, non-intuitive impact function, have been put forward by Tóth et al. (2011), Mastromatteo et al. (2014a, 2014b) and Farmer et al. (2013) (on a related topic, see also Taranto et al., 2014).

Still, whereas the description of the contemporaneous impact of orders in terms of Eq. (1) is by now well accepted, the fate of this impact after the order has been completed is still not settled and is a matter of debate. On general grounds, one expects this impact to relax from its peak value, since upon 
conditioning on the fact that the extra buyer (seller) is no longer active, the subsequent flow is biased in the opposite direction. This is clearly the case in the "latent order book" model and its extensions (Tóth et al., 2011; Mastromatteo et al., 2014a; Donier et al., 2014), but has also been argued on theoretical grounds by Farmer et al. (2013) (see also Donier, 2012). Based on fair pricing (no arbitrage) arguments, Farmer et al. (2013) propose that the asymptotic value of the impact (long after the order has been completed) should be a fraction of the peak value, in such a way that the average price paid by the buyer (seller) is equal to the long-term price. This would imply zero average profit both for the liquidity provider and for the trader. For a concave impact and a constant rate of execution, it is easy to derive from Eq. (1) that the average paid price is $\theta /(1+\delta)$ above the initial price $p(0)$. Therefore, the long-time plateau value of the impact is predicted to be $2 / 3$ of the peak value when $\delta=1 / 2$. This prediction appears to be borne out by two empirical papers (see Moro et al., 2009; Bershova and Rakhlin, 2013), while a third study (see Gomes and Waelbroeck, 2014) reports a more complex picture, where "informed" trades and "uninformed" trades lead to a very different impact relaxation pattern. In the former case, the impact seems to relax towards the predicted $2 / 3$ value, while in the latter case, impact appears to relax all the way to zero. Farmer et al. (2013) and Gomes and Waelbroeck (2014) argue that uninformed trades should have no long-term impact, and that the long-term impact of informed trades should reflect, on an average, the information content of these trades. Incidentally, both papers (see Bershova and Rakhlin, 2013; Gomes and Waelbroeck, 2014) confirm once again the validity of square-root impact law, Eq. (1).

While we agree with the general ideas expressed by Gomes and Waelbroeck (2014), there are several issues that need to be clarified. First, from a theoretical point of view - if some trades are uninformed and others are informed, the simple fair pricing argument of Farmer et al. (2013) and Donier (2012) becomes more subtle since liquidity providers can make money out of "noise traders", as in Kyle's model (Kyle, 1985). Second, as recognized by Gomes and Waelbroeck (2014), the notion of informed trades is at best ambiguous and needs to be supplemented by a time horizon, over which the prediction is supposed to be realized. If buying a stock is with the objective of very short-term gains, on the same time scale as the measurement of impact relaxation, then the distinction between informed and uninformed trades may indeed be warranted. But if the future gain is expected over several months while impact is measured over a few hours to a few days (beyond which the noise level becomes insuperable), then clearly the distinction is 


\section{Brokmann et al.}

moot. Third, and perhaps most importantly, there is a methodological problem with the way the data has been analyzed in all previous work on the subject. The point is that proprietary order-flows tend to be highly autocorrelated in time (due to the slicing of large buy/sell orders over several days or sometimes weeks). The price dynamics seen after the completion of a day long meta-order (that may well be part of a longer meta-order) therefore reflects both its own decaying impact and the impact dynamics from all previous and following orders as well. This requires some deconvolution treatment that turns out to be crucial to properly address the question of impact decay — as the empirical and theoretical analysis below clearly demonstrates.

In this paper, we carefully analyze the impact of CFM's proprietary trades on equity markets. We show that our data is, after deconvolution, compatible with a price model where the "mechanical" impact of meta-orders decays all the way to zero, superimposed with some (investor-dependent) predictability pattern. In other words, we posit that the average price profile at time $t$ after the completion time $T$ of a single meta-order, of volume $Q$ and predictability $\alpha$, is the sum of two fundamentally different contributions ${ }^{1}$ :

$$
\mathbb{E}_{0}[p(t>T)] \approx p(0)[1+\theta(Q) I(t-T)+\alpha H(t)] .
$$

In the above equation, $\theta(Q) \equiv \epsilon(Q) Y_{0} \sigma[Q / V]^{\delta}$ is the instantaneous impact of the trade, $I(\tau)$ is a "propagator" describing the mechanical part of the impact (Bouchaud et al., 2004), which decreases from $I(0)=1$ to $I(\infty) \approx 0$. The predictor amplitude $\alpha$ (possibly equal to zero for uninformed trades) is the expected long-term price profile that motivated the trade at time $t=0$ and $H(t)$ describes the way this prediction realizes itself over time. For example, for an Ornstein-Uhlenbeck predictor, $H(t)=1-\exp (-\Gamma t)$ for a prediction on a horizon $\sim \Gamma^{-1}$ (see below). For uninformed trades, or for bets on very long horizons such that $\Gamma t \ll 1$, one can set $\alpha=0$. This, we believe, allows one to stitch together various aspects of the literature. However, as we shall argue in the conclusion, the model defined by Eq. (2) is not entirely consistent, since it is quasi-linear (different meta-orders add up linearly) whereas the concavity of the impact of each single meta-order (Eq. (1)) indicates strong nonadditive behavior, at least on short time scales. A fully consistent theory that allows one to deal with nonadditive, time-dependent impact can

\footnotetext{
${ }^{1}$ This decomposition is also assumed in Almgren et al. (2005) and Farmer et al. (2013), and can in fact be formally justified within the latent order book model, see the recent paper of Donier et al. (2014).
} 
be found in Donier et al. (2014) and suggests that Eq. (2) should in fact be a reasonable approximation.

\section{An Illustrative Toy-Model}

Let us first present a toy-model that highlights the difference between the "true" impact $I(\tau)$ and the impact of a sequence of correlated trades, resulting from an investor who attempts to optimize his gains. We consider an idealized market where impact is linear and decays instantaneously, i.e., the true impact is $I(\tau)=\gamma \delta(\tau)$. We imagine that the investor has an Ornstein-Uhlenbeck normalized signal $s(t)$ with correlation time $\Gamma^{-1}$, such that

$$
\langle s(\tau) s(0)\rangle=e^{-\Gamma|\tau|} .
$$

The prediction based on this signal is that the price change between $t$ and $t+d t$ is

$$
\left\langle p_{\mathrm{d}}(t+d t)-p_{\mathrm{d}}(t) \mid s(t)\right\rangle=\mathcal{G} s(t) d t,
$$

where $\mathcal{G}$ sets the scale of the predicted gain, while the integrated expected gain up to time $t+\tau$ is

$$
\left\langle p_{\mathrm{d}}(t+\tau)-p_{\mathrm{d}}(t) \mid s(t)\right\rangle=s(t) \frac{\mathcal{G}}{\Gamma}[1-\exp (-\Gamma \tau)] .
$$

The investor wants to optimize the total gain, impact costs included, for a fixed level of risk $R^{2}$. This is implemented (in continuous time) as the maximization of

$$
\mathcal{L}=\frac{1}{T}\left[\int_{0}^{T} d t \pi(t) \mathcal{G} s(t)-\frac{\gamma}{2} \int_{0}^{T} d t \dot{\pi}(t)^{2}-\lambda \int_{0}^{T} d t \pi(t)^{2}\right],
$$

where $\pi(t)$ is the position (in $\$$ ) at time $t$ and $\gamma$ is the impact coefficient: Trading at speed $\dot{\pi}$ pushes the price instantaneously by an amount $\gamma \dot{\pi}$. The average price paid in the process is $\frac{1}{2} \gamma \dot{\pi}$. $\lambda$ is the Lagrange multiplier allowing one to fix the average risk at a chosen value $R^{2}$. We assume that $T \rightarrow \infty$ in the following and forget about the bounds on $t$.

The above quadratic optimization problem can easily be solved (see, e.g., Henry, 2007; Garleanu and Pedersen, 2013). The solution of this problem is to fix the position $\pi(t)$ proportional to an exponential moving average of the predictor:

$$
\pi(t)=\phi_{0} \int_{-\infty}^{t} d t^{\prime} e^{-\omega\left(t-t^{\prime}\right)} s\left(t^{\prime}\right), \quad \phi_{0}=\frac{\mathcal{G}}{\gamma(\omega+\Gamma)},
$$




\section{Brokmann et al.}

where the averaging frequency $\omega$ is set by the following equation:

$$
z(1+z)^{3}=\frac{\sigma^{2} \mathcal{G}^{2}}{\gamma^{2} R^{2} \Gamma^{4}}, \quad \omega=z \Gamma .
$$

As expected, $z \rightarrow \infty$ when $\gamma \rightarrow 0$, i.e., the position tracks instantaneously the signal. As the friction becomes higher however, the signal has to be slowed down in order to extract a positive gain from the signal.

Now, we want to compute the measured impact induced by this optimal trading policy. More precisely, we will define the "raw" impact $I_{\text {raw }}(\tau)$, normalized by the average price impact due to trading at the end of the first trading period (i.e., at $t+d t$, just before the "true" impact decays back to zero), as

$$
\left\langle\left(p_{\mathrm{d}}(t+\tau)-p_{\mathrm{d}}(t)\right) \dot{\pi}(t)\right\rangle=\gamma\left\langle\dot{\pi}(t)^{2}\right\rangle \times I_{\text {raw }}(\tau), \quad(\tau>0) .
$$

This is the impact one would measure by averaging the average price profile over all the trades of the investor, without any deconvolution (hence the term "raw"). It is easy to show that

$$
\left\langle\left(p_{\mathrm{d}}(t+\tau)-p_{\mathrm{d}}(t)\right) \dot{\pi}(t)\right\rangle=\gamma \phi_{0}^{2}[1-\exp (-\Gamma \tau)]
$$

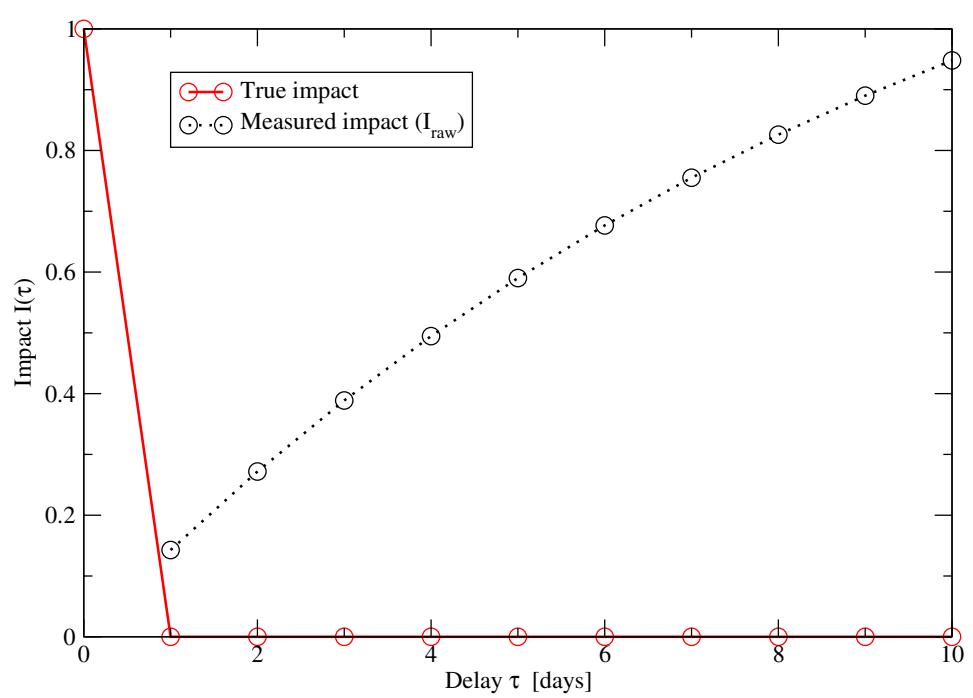

Fig. 1. Illustration of the difference between the "true" impact $I(\tau)$ of an isolated order (here chosen to be a $\delta$-function in time) and the apparent impact $I_{\text {raw }}(\tau)$ of a series of correlated trades, resulting from optimizing the expected gain of a strategy based on an OrnsteinUhlenbeck predictor, with $\Gamma=0.1$ and $z=0.5$. 
which has to be normalized by

$$
\gamma\left\langle\dot{\pi}(t)^{2}\right\rangle=\frac{1}{(z+1)} \gamma \phi_{0}^{2},
$$

finally leading to (see Fig. 1)

$$
I_{\text {raw }}(\tau)=(1+z)[1-\exp (-\Gamma \tau)] .
$$

This calculation reveals that although $I(\tau)$ is a $\delta$-function in time, $I_{\text {raw }}(\tau)$ increases from zero to a nonuniversal plateau value $=1+z$ which depends continuously on the a-dimensional variable $\gamma R \Gamma^{2} / \sigma \mathcal{G}$ that measures the relative strength of the impact costs and the expected gains. ${ }^{2}$

\section{Empirical Data and Impact Decay}

The above toy-model clearly shows that in order to measure the "true impact" $I(\tau)$, a careful deconvolution of the raw impact must be performed. Our empirical analysis is based on a proprietary CFM dataset, consisting in the exhaustive list of 1.6 million "meta-orders" (defined below), together with the daily signals that lead to these trades, over three full years (2011-2013) in a variety of equity markets (Europe, US, Japan and Australia), corresponding to meta-orders on roughly 2000 different stocks per day. Trading decision are taken once a day on the basis of a signal $\{s(0), s(1), \ldots$, $s(t), s(t+1), \ldots\}$, resulting in a sequence signed quantities $\{q(0), q(1), \ldots$, $q(t), q(t+1), \ldots\}$ (where $q(t):=\epsilon(t) Q(t)$ ) that we call meta-orders. ${ }^{3}$

Each meta-order is to be executed throughout the day (from open to close), starting at decision midpoint prices $\left\{p_{\mathrm{d}}(0), p_{\mathrm{d}}(1), \ldots, p_{\mathrm{d}}(t), p_{\mathrm{d}}(t+\right.$ $1), \ldots\}$ and executed at average prices $\left\{p_{\mathrm{x}}(0), p_{\mathrm{x}}(1), \ldots, p_{\mathrm{x}}(t), p_{\mathrm{x}}(t+1), \ldots\right\}$. Typical volume fractions are in the range $Q / V \sim 0.1-5 \%$ (see Fig. 2). Note that meta-orders, when started, are always (nearly) fully executed, with an average execution rate exceeding $99 \%$. There is therefore no selection bias in the execution price of our trades. This is extremely important, since any correlation between the execution rate and the market direction would induce important biases in impact measurements (for example, such correlations can lead to a negative measured impact).

The daily signals $\{s(0), s(1), \ldots, s(t), s(t+1), \ldots\}$ correspond to CFM's best prediction of future prices, and are a combination of a variety of medium

\footnotetext{
${ }^{2}$ Note in particular that the "fair value" advocated in Farmer et al. (2013) (corresponding, with the present normalization, to $I=1 / 2$ ) does not seem to play any special role here.

${ }^{3}$ Note that this definition is different from that used in Farmer et al. (2013), where a metaorder is the aggregate of all orders posted by investors sharing the same information signal.
} 


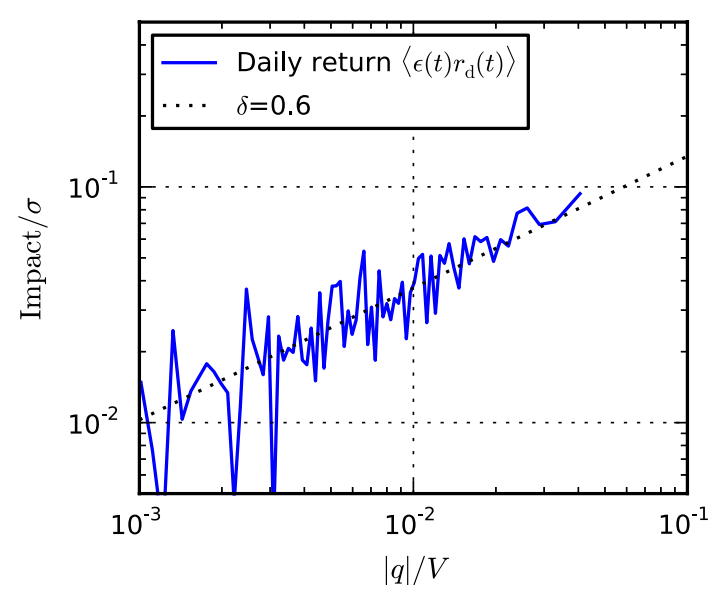

Fig. 2. Impact as a function of the volume fraction $|q| / V$ of traded meta-orders on equity markets. The line is the average of the impact for a given binned value of $|q| / V$. The dependence is concave and follows a power-law scaling of exponent $\delta=0.6$ over the whole range of $|q| / V$. This value of $\delta$ turns out to be exactly the same as the one obtained by Almgren et al. (2005).

to long-term technical and fundamental indicators, and with a prediction horizon $\Gamma^{-1}$ ranging from 10 days to 100 days. The signals $s(t)$ are computed just before the execution of the meta-order and are normalized to have unit variance. The signals are furthermore made market neutral so all results reported below are, to first-order, immune to market directional effects.

Due to price impact, the execution prices $p_{\mathrm{x}}(t)$ are on average higher (lower) than the initial market midpoint $p_{\mathrm{d}}(t)$ when the buy (sell) meta-order started, causing both the "strike slippage" $r_{\mathrm{x}}(t)=\left(p_{\mathrm{x}}(t)-p_{\mathrm{d}}(t)\right) / p_{\mathrm{d}}(t)$ and the price change $r_{\mathrm{d}}(t)=\left(p_{\mathrm{d}}(t+1)-p_{\mathrm{d}}(t)\right) / p_{\mathrm{d}}(t)$ between two daily trading decisions to exhibit the expected square-root dependence on $Q / V$ (see Fig. 2, where the fitting line corresponds to $\delta=0.6$ ), in agreement with Eq. (1) and well in line with all previous research (see in particular (Almgren et al., 2005)).

We now turn to measuring the decay of impact over subsequent days. We first define the raw average impact $I_{\text {raw }}(\tau)$ through the following regression:

$$
\frac{p_{\mathrm{d}}(t+\tau)-p_{\mathrm{d}}(t)}{p_{\mathrm{d}}(t)}=I_{\text {raw }}(\tau) \theta(t)+\xi(t)
$$

where $\xi$ is a noise term, and again $\theta(t) \equiv Y_{0} \epsilon(t) \sigma[Q(t) / V]^{\delta}$. Note that by definition, $I_{\text {raw }}(\tau)$ is normalized to unity for $\tau=1^{-}$, i.e., at the end of the meta-order of the first day (as for our toy-model above). We also define, in the same units, the average cost $I_{\mathrm{x}}$ incurred by our execution, as caused by the impact of this order on $p_{\mathrm{x}}(t)$ and any other micro-structural effects: 

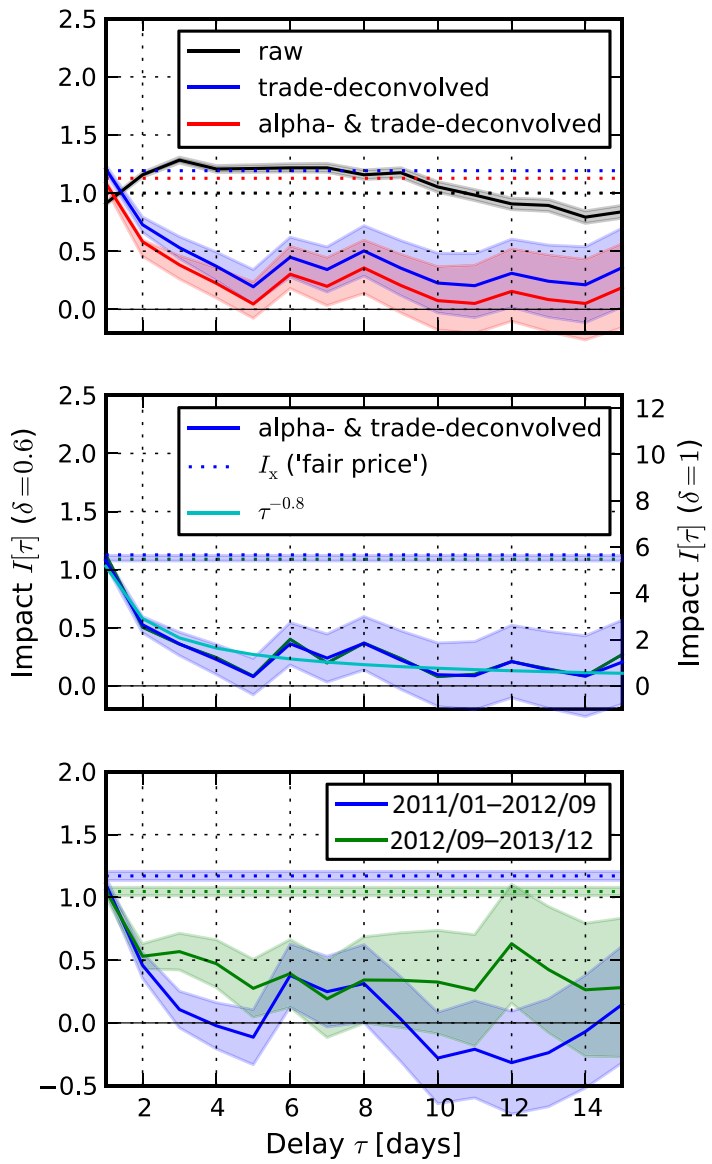

Fig. 3. Impact dynamics, obtained through the regression Eq. (13), averaged over all trades, markets and periods with an equal weight, normalized by the execution price $I_{\mathrm{x}}$. The shaded region corresponds to 2- $\sigma$ error bars from the standard error of the Ordinary Least-Square (OLS) regression. The lag is counted in business days. (a) Raw impact appears to reach a plateau for the raw data (black line) but true impact is found to be decaying (blue/red lines) once returns are corrected from the influence of past trades and predictability. The price reverts to its pre-trade value, or at least well below the average the execution price (horizontal dotted line at $y=1$ ). (b) Impact deconvolved profiles for $\delta=0.6$ (green) and $\delta=1$ (blue) and simple power-law fit, yielding $I(\tau) \propto \tau^{-0.8}$, to be compared with the theoretical prediction of Donier et al. (2014): $I_{\text {th. }}(\tau) \propto \tau^{-1 / 2}$. (c) Impact decay profiles as measured over two disjoint time periods. Colored figure is available for the electronic version of the article.

$r_{\mathrm{x}}(t)=I_{\mathrm{x}} \theta(t)$. The result of the regression Eq. (13) averaged over all markets and all periods is shown as the roughly constant black line in Fig. 3(a). Note that in this graph we have divided $I_{\text {raw }}(\tau)$ by $I_{\mathrm{x}}$, in such a way that "fair pricing" should yield a horizontal line $y=1$. One sees that, in apparent agreement with the fair-price argument, the (rescaled) price indeed remains 


\section{Brokmann et al.}

in the vicinity of the executed price $I_{\mathrm{x}}$ several days after the meta-order was completed.

However, the autocorrelation $\langle\epsilon(t) \epsilon(t+\tau)\rangle$ of the sign of the trades is found significantly positive over several days, obfuscating, as illustrated by our toy-model above, how much of the raw dynamics $I_{\text {raw }}(\tau)$ actually reflects the impact of a single meta-order. In other words, the impact of the trades following the initial one keeps the impact $I_{\text {raw }}(\tau)$ artificially high. Furthermore, since the trades are initiated in part by predictive signals, one should expect this signal to reveal itself through time and contribute to $I_{\text {raw }}(\tau)$.

Instead of temporally aggregating successive meta-orders of the same sign as in Bershova and Rakhlin (2013) (a noncausal procedure which induces conditioning artefacts when a new decision is taken each day), or using a somewhat ad hoc correction to compensate for the trades occurring during the decay of impact (Gomes and Waelbroeck, 2014), we propose to deconvolve jointly past trades and predictions from the raw impact dynamics assuming a quasi-linear model, i.e., linearly regressing the daily returns $r_{\mathrm{d}}(t)$ on both past impacts $\{\theta(t), \theta(t-1), \ldots\}$ and return predictions $\{s(t), s(t-1), \ldots\}$, exploiting the ability of multiple least-square regression to properly account for all correlations among regressors:

$$
r_{\mathrm{d}}(t)=\sum_{t^{\prime}=t-M}^{t} G_{\theta}\left(t-t^{\prime}\right) \theta\left(t^{\prime}\right)+\sum_{t^{\prime}=t-M}^{t} G_{s}\left(t-t^{\prime}\right) s\left(t^{\prime}\right)+\xi(t),
$$

where $M$ is a certain maximum lag that does not affect much our conclusions provided it is taken to be larger than five days. In fact, $G_{s}\left(t-t^{\prime}\right)$ is found to be close to $G_{s}(0) \delta_{t, t^{\prime}}$, as expected when the predictor is itself optimal. Instrumental aspects to successfully derive the kernels $G_{\theta, s}(\tau)$ include: (i) working on a complete set of proprietary trades - given the potential of incomplete datasets to exhibit detrimental bias in the trade autocorrelation estimation - and (ii) performing the linear regression on the different market zones (EU, US, Japan and Australia) separately to ensure maximal homogeneity within the dataset — as required in any basic least-square deconvolution.

From the determination of $G_{\theta}(\tau)$, the "true" impact kernel $I(\tau)$ (stripped out of the contribution of both predictions and correlated trades) can be reconstructed as $I(\tau)=\sum_{t^{\prime}=0}^{\tau} G_{\theta}\left(t^{\prime}\right)$. The average returns during meta-order execution $r_{\mathrm{x}}(t)$ are treated similarly to compute the corresponding $\left\{G_{\theta, \mathrm{x}}\left(t^{\prime}\right)\right\}_{t^{\prime} \in[0, M]}$, with $G_{\theta, \mathrm{x}}(0)$ providing an execution price proxy itself deconvolved from past trades and predictions. 
Figure 3(a) illustrates the result of this approach to deconvolve $I_{\text {raw }}(\tau)$ from either past trades only (i.e., enforcing $G_{s}(t) \equiv 0$ in Eq. (14)), or both past trades and predictors. One can see from Fig. 3(a) that the main effect in fact comes from the correlation of meta-orders, which, when taken into account, leads to a significant decay of $I(\tau)$, much below the execution price $I_{\mathrm{x}}$ (blue line). Removing the prediction signal decreases further the impact, which decays to a very small value beyond 10 days or so (red line). The difference between the red and the blue line is however small, meaning that the average prediction strength of the signal $s(t)$ is small on short-term horizons (in line with the fact that $\Gamma^{-1} \sim 10-100$ days). The decay profiles are also independent on the exact value of the impact exponent $\delta$ that is used

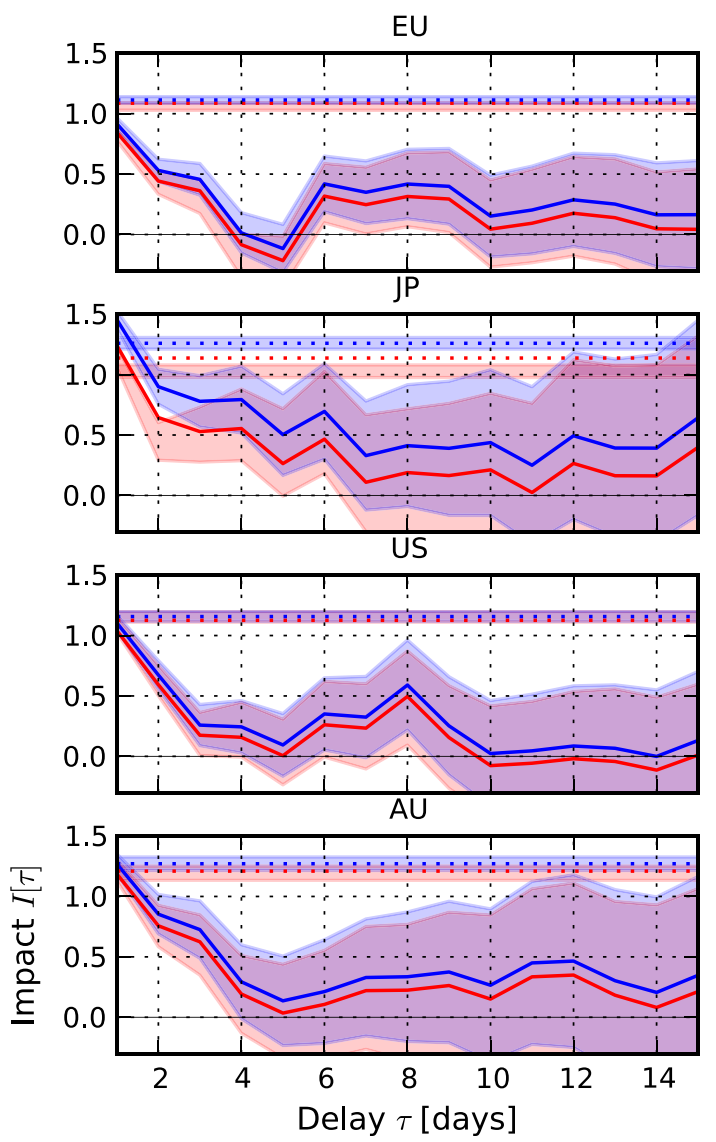

Fig. 4. Impact dynamics for different equity markets (here EU, US, Australia and Japan), once deconvolved of the influence of past trades (red) and predictability (blue) and normalized by the average execution price $I_{\mathrm{x}}$. Colored figure is available for the electronic version of the article. 
to define $\theta(t)$. Figure 3(b) shows that the decay profile remains essentially unchanged for $\delta$ ranging from 0.5 (square-root impact) to 1 (linear impact) - providing strong evidence that the observed decay of $I(\tau)$ is a genuine property of impact dynamics rather than a deconvolution artefact. A simple power-law fit suggests a slow decay $I(\tau) \sim \tau^{-0.8}$, similar to the decay of impact of individual trades (see Bouchaud et al., 2004, 2006), and close to the theoretical prediction of Donier et al. (2014): $I_{\text {th. }}(\tau) \propto \tau^{-1 / 2}$. Deconvolved profiles appear reasonably stable both in time (Fig. 3(c)) and across equity markets (Fig. 4). Other minor variations are due to, e.g., unresolved seasonality patterns less amenable to our multiple least-square regression approach.

\section{Discussion and Conclusion}

The strong divergence between raw and deconvolved impact decay profiles shown in Fig. 3(a) highlights the subtle interplay between trades, their information content and price dynamics. Once past trades and predictable returns are removed, our results suggest (in agreement with the assumptions made in Almgren et al. (2005), Donier et al. (2014) and Farmer et al. (2013)) that:

(i) The price dynamics can be decomposed into a "mechanical" impact $I(\tau)$, identical for all trades (as expected on anonymous markets) and an information part;

(ii) the mechanical impact $I(\tau)$ of an isolated, uninformed meta-order appears to be fully transient and decays all the way to zero, albeit quite slowly (see Figs. 3(a) and 3(b) and the work of Gomes and Waelbroeck (2014)). ${ }^{4}$

We therefore believe that the previously reported asymptotic " $2 / 3$ " level of impact decay is likely to be caused by the impossibility of adequately subtracting information and trade correlations from the data - echoing previous comments in Gomes and Waelbroeck (2014). Furthermore, the relevance of the simple fair-price argument proposed in Farmer et al. (2013) is not clear to us since, as highlighted by our toy-model above, there is no direct relation between the size of an order and its information content.

\footnotetext{
${ }^{4}$ See the recent discussion in A. Alfonsi and P. Blanc, Dynamic Optimal Execution in a MixedMarket-Impact Hawkes Price Model, arXiv:1404.0648 [q-fin.TR], where the asymptotic value of the impact $I(\infty)$ is shown to be related to the fraction of endogenously generated trades $n$ through $I(\infty)=1-n$. Therefore, a low value of $I(\infty)$ is compatible with the value $n \approx 1$ reported in the Hawkes analysis of Hardiman et al. (2013) and Hardiman and Bouchaud (2014) and references therein.
} 
There are still a number of open questions that future research, both theoretical and empirical, should try to address. In particular, our approach explicitly assumes impact decay to be a quasi-linear time-invariant process (Eq. (14)), which is in fact Gatheral's model in discrete time (Gatheral, 2010; see also Bouchaud et al., 2004). This is a seemingly plausible assumption given the consistency of the results presented so far, but in contradiction with the square-root law itself, which signals that impact is not additive. A recent theory that captures both nonadditive and time-dependent impact (Donier et al., 2014) suggests that the approximations made in Eq. (14) above are in fact quite reasonable. Still, we have seen in our data set hints of nonlinear effects. For example, the impact decay $I(\tau)$ measured on the sub-sample of trades that correspond to the top-half participation rate over the last 10 days is noticeably different from the one measured on the bottom-half (the former decaying faster than the latter). This of course should not be the case within the above quasi-linear description. Furthermore, concavity and time decay are not easy to disentangle. For example, if aggregated meta-orders were still in the concave square-root regime over five days, this would lead to an $a p$ parent decay of $\approx 60 \%$ of impact when fitted by a quasi-linear model, even if impact was fully permanent. ${ }^{5}$ We have however checked that our results are essentially unchanged when regrouping two successive days in a new elementary time unit and redoing the above analysis, suggesting that time decay indeed dominates over persistent concavity for $\tau>1$ day.

Finally, from a purely methodological point of view, our OLS regression is most certainly suboptimal. Although the above regressions were always found to nonsingular, there might be uncontrolled biases that need to be better understood. We plan to study this issue in details in the near future.

\section{Acknowledgments}

We thank R. Bénichou, P. Blanc, J. Bonart, J. De Lataillade, J. Donier, Z. Eisler, D. Farmer, J. Gatheral, S. Hardiman, E. Henry, L. Laloux, C.-A. Lehalle, F. Lillo, Y. Lempérière, I. Mastromatteo, M. Potters, D. Taranto, B. Tóth, H. Waelbroeck and E. Zarinelli for many useful conversations on these issues.

\section{References}

Almgren, R., C. Thum, E. Hauptmann and H. Li, 2005, Direct Estimation of Equity Market Impact, Risk 18(7), 57-62.

\footnotetext{
${ }^{5} \mathrm{R}$. Benichou and Y. Lempérière, private communication.
} 
Bénichou, R. and Y. Lempérière, private communication.

Bershova, N. and D. Rakhlin, 2013, The Non-Linear Market Impact of Large Trades: Evidence from Buy-Side Order Flow, Quantitative Finance 13, 1759-1778.

Bladon, A. J., E. Moro and T. Galla, 2013, Agent-Specific Impact of Single Trades in Financial Markets, Physical Review E 85, 036103.

Bouchaud, J. P., J. Farmer and F. Lillo, 2008, How Markets Slowly Digest Changes in Supply and Demand, in T. Hens and K. Schenk-Hoppe (eds.), Handbook of Financial Markets: Dynamics and Evolution, pp. 57-156, Elsevier: Academic Press, Massachusetts.

Bouchaud, J.-P., J. Kockelkoren and M. Potters, Random Walks, Liquidity Molasses and Critical Response in Financial Markets, Quantitative Finance 6, 115-123.

Bouchaud, J. P., Y. Gefen, M. Potters and M. Wyart, 2004, Fluctuations and Response in Financial Markets: The Subtle Nature of "Random" Price Changes, Quantitative Finance 4(2), 176-190.

Donier, J., 2012, Market Impact with Autocorrelated Order Flow under Perfect Competition, arXiv:1212.4770 [q-fin.TR].

Donier, J., J. Bonart, I. Mastromatteo and J.-P. Bouchaud, 2014, A Fully Consistent, Minimal Model for Non-Linear Market Impact, arXiv:1412.0141 [q-fin.TR].

Farmer, J. D., A. Gerig, F. Lillo and H. Waelbroeck, 2013, How Efficiency Shapes Market Impact, Quantitative Finance 13, 1743-1758.

Garleanu, N. and L. H. Pedersen, 2013, Dynamic Trading with Predictable Returns and Transaction Costs, The Journal of Finance 68, 2309-2340.

Gatheral, J., 2010, No-Dynamic-Arbitrage and Market Impact, Quantitative Finance 10, 749-759.

Gomes, C. and H. Waelbroeck, 2014, Is Market Impact a Measure of the Information Value of Trades? Market Response to Liquidity vs. Informed Trades, Quantitative Finance 15(5), 773-793.

Hardiman, S. J., N. Bercot and J. P. Bouchaud, 2013, Critical Reflexivity in Financial Markets: A Hawkes Process Analysis, The European Physical Journal $B 86,1-9$.

Hardiman, S. J. and J. P. Bouchaud, 2014, Branching Ratio Approximation for the Self-Exciting Hawkes Process, Physical Review E90, 062807.

Henry, E., 2007, Optimal Slowing Down of a Predictor, CFM Internal Report, unpublished.

Kyle, A., 1985, Continuous Auctions and Insider Trading, Econometrica 53, 13151335.

Kyle, A. P. and A. Obizhaeva, 2012, Large Bets and Stock Market Crashes, available at SSRN 2023776.

Mastromatteo, I., B. Tóth and J. P. Bouchaud, 2014a, Agent-Based Models for Latent Liquidity and Concave Price Impact, Physical Review E 89, 042805.

Mastromatteo, I., B. Tóth and J. P. Bouchaud, 2014b, Anomalous Impact in Reaction-Diffusion Models, Physical Review Letters 113, 268701.

Moro E., J. Vicente, L. G. Moyano, A. Gerig, J. D. Farmer, G. Vaglica, F. Lillo and R. N. Mantegna, 2009, Market Impact and Trading Profile of Hidden Orders in Stock Markets, Physical Review E 80, 066102. 
Taranto, D. E., G. Bormetti and F. Lillo, 2014, The Adaptive Nature of Liquidity Taking in Limit Order Books, Journal of Statistical Mechanics, P06002.

Torre, N., 1997, BARRA Market Impact Model Handbook, BARRA Inc., Berkeley. Tóth, B., Y. Lempérière, C. Deremble, J. De Lataillade, J. Kockelkoren and J. P. Bouchaud, 2011, Anomalous Price Impact and the Critical Nature of Liquidity in Financial Markets, Physical Review X 1(2), 021006.

Zarinelli, E., M. Treccani, J. D. Farmer and F. Lillo, 2014, Beyond the Square Root: Evidence for Logarithmic Dependence of Market Impact on Size and Participation Rate, arXiv:1412.2152. 